\title{
Auch kleine Eichhörnchen können zusammen grosse Nüsse tragen*
}

\author{
Paula Bezzola ${ }^{a}$, \\ Anna Mascherek ${ }^{b}$ \\ a Stv. Geschäftsführerin Stiftung \\ für Patientensicherheit \\ b Wissenschaftliche \\ Mitarbeiterin Stiftung \\ für Patientensicherheit
}

* Nach einem Sprichwort aus Kamerun
Korrespondenz:

Paula Bezzola

Stiftung für Patientensicherheit Asylstrasse 77

CH-8032 Zürich

Tel. 0432441480

Fax 0432441481

info[at]patientensicherheit.ch
Im Rahmen des Pilotprogramms progress! Patientensicherheit Schweiz bietet die Stiftung für Patientensicherheit für die Schweiz neuartige, umfassende Vertiefungsprojekte zur Erhöhung der Patientensicherheit an. Innerhalb von zwei Jahren streben Pilotbetriebe in einem strukturierten Rahmen gemeinsam klare Ziele an. Die im Programm vorgesehenen Aktivitäten und Massnahmen passen die Betriebe je nach betrieblicher Ausgangslage und Gegebenheiten an. Die Zielerreichung wird mit gemeinsamen Evaluationserhebungen evaluiert. Das erste Vertiefungsprojekt progress! Sichere Chirurgie strebt die erfolgreiche, umfassende Implementierung der chirurgischen Checkliste für Schweizer Betriebe an.

\section{"Quality Improvement Collaboratives», bzw. «Large Scale Initiatives» - ein Patentrezept?} Der Gesundheitsversorgung mangelt es nicht an gutevaluierten und wirksamen Interventionen, um die Patientensicherheit zu verbessern. So ist beispielsweise die Wirksamkeit von Massnahmen zur Reduktion von Infektionen bei der Legung von Kathetern [1] oder die der Anwendung chirurgischer Checklisten zur Reduktion von unerwünschten Ereignissen im OP [2] gut dokumentiert. Die Umsetzung dieser Interventionen ist allerdings oft nicht zufriedenstellend. So bleiben Patienten in der Realität Massnahmen verwehrt, obwohl diese in der Literatur als wirksam beschrieben werden [3]. Die Diskrepanz zwischen dem, was theoretisch möglich wäre, und dem, was in der Praxis zum Standard gehört, ist gross. Die Implementierung neuer Standards ist nicht nur abhängig von der Akzeptanz der Massnahme bei den einzelnen Mitarbeitenden, sondern ist vor allem auch eine systemische Herausforderung, bei der alle Ebenen einer Organisation einbezogen und beteiligt sein müssen [4]. In der Praxis sind Personen deswegen meist weniger mit der Frage konfrontiert, welche Massnahme eingeführt werden soll, sondern vor allem mit der Frage, wie diese umgesetzt werden kann (vgl. [3]). «Quality Improvement Collaboratives», bzw. « Large Scale Initiatives» bieten strukturierte Programme für mehrere Organisationen oder für gesamte Landesteile an, um Massnahmen zur Förderung der Patientensicherheit und Erhöhung der Qualität in der Gesundheitsversorgung erfolgreich umzusetzen. Die grundlegende Idee dieser Programme besteht in der interdisziplinären Zusammenarbeit von Teams aus verschiedenen Gesundheitsbereichen oder -organisationen, die sich über einen bestimmten Zeitraum hinweg zusammenschliessen und gemeinsam versuchen, Massnahmen strukturiert umzusetzen [5]. Mit dem gemeinsamen Vorgehen und der Fokussierung auf ein spezifisches Problem und ausgewählten Massnahmen gewinnen die betriebsinternen Projekte an Gewicht, und deren Befürworter werden in ihrem Veränderungsprozess bestärkt. Die Entwicklung, der Aufbau und die Koordination des Programms auf zentraler Ebene ermöglichen ein effizientes, ressourcenschonendes Vorgehen. Auch wenn einige Studien den positiven Effekt solcher Programme durchaus zeigen, bleibt neben der praktischen Durchführung auch die wissenschaftliche Evaluation zur Erforschung der Wirkmechanismen eine Herausforderung [5-7]. Einerseits liegt dies an der Schwierigkeit, Studien zu designen, die die Komplexität einer ganzen Organisation berücksichtigen können, andererseits an der Heterogenität der Interventionen und der beteiligten Organisationen an sich, die eine Vergleichbarkeit schwierig machen [5, 8]. Zudem erfolgen in der Regel parallel zu den Programmen laufend Veränderungen im Gesamtsystem, die ebenfalls Einfluss auf die gewünschte Wirkung des Programms haben können. Bis heute gibt es also kein «Patentrezept», nach dem wirksame Massnahmen für die Patientensicherheit auch erfolgreich implementiert und zum Standard gemacht werden können, so dass alle Patienten routinemässig von diesen wirksamen Massnahmen profitieren können. Es besteht aber Konsens in der Literatur darüber, dass neben dem strukturierten, gemeinschaftlichen und interprofessionellen Vorgehen weitere grundlegenden Faktoren sowohl auf der strategischen Ebene als auch auf der Ebene der praktischen Umsetzung wichtig sind: das Engagement und die Verpflichtung der oberen Führungsetagen, die verbindliche Einbindung und Beteiligung aller Mitarbeitenden, die Bereitstellung ausreichender zeitlicher, finanzieller und inhaltlicher Ressourcen, die sorgfältige Planung und Umsetzung der Massnahmen, praktisches Training der Massnahmen vor Ort, die Vernetzung mehrerer Betriebe zum Wissensaustausch und das Vorhandensein oder die Erarbeitung einer unterstützenden, fördernden Betriebskultur. Ein weiterer wichtiger Erfolgsfaktor sind aussagekräftige, auf das Programm abgestimmte Evaluationserhebungen, die eine hohe Datenqualität aufweisen [3, 5-9]. 


\section{Realisierung gemeinschaftlicher strukturier- ter Pilotprogramme für Schweizer Betriebe}

Das Problem der Diskrepanz zwischen theoretisch vorhandenen Massnahmen und deren konsequenter Umsetzung in der Praxis stellt sich auch konkret für die Schweiz. Die Stiftung für Patientensicherheit führt nun deshalb im Rahmen der nationalen Qualitätsstrategie des Bundes und unter massgeblicher Finanzierung des Bundesamtes für Gesundheit in den nächsten Jahren zwei nationale Pilotprogramme unter dem Namen progress! Sichere Patientensicherheit Schweiz durch. Als erstes Pilotprogramm wurde progress! Sichere Chirurgie lanciert, als zweites wird progress! Sichere Medikation an Schnittstellen folgen ${ }^{1}$. Beide Programme bieten neben einer nationalen Verbreitung von Empfehlungen und Hintergrundinformationen ein Vertiefungsprojekt für ausgewählte interessierte Betriebe an. Diese Vertiefungsprojekte lehnen sich an die oben beschriebenen Erfolgsfaktoren der «Quality Improvement Collaboratives», bzw. «Large Scale Initiatives» an, sind an die Ausgangslage des schweizerischen Gesundheitswesen angepasst und werden aufgrund der Erkenntnisse aus bisherigen Qualitätsprojekten in der Schweiz konzipiert. Diese umfassende Art von Vertiefungsprojekten wird in der Schweiz erstmalig angeboten.

\section{Konzept und Angebot des Vertiefungsprojekts progress! Sichere Chirurgie}

Die Anwendung der interprofessionellen chirurgischen Checkliste ist eine konkrete Massnahme, deren Wirksamkeit zur Reduktion von unerwünschten Ereignissen in der Chirurgie erwiesen ist. Auch wenn die Anwendung der chirurgischen Checkliste auf den ersten Blick in der Schweiz schon recht verbreitet ist, besteht bei der Umsetzung Handlungsbedarf - auch dort, wo bereits eine Checkliste angewendet wird ${ }^{2}$. Für die Wirksamkeit der Checkliste ist jedoch eine konsequente, richtige und 100\%-ige Anwendung bei
Vorgehen die gewünschte Erhöhung der Compliance der korrekten Anwendung der chirurgischen Checkliste erreicht werden. Der Fokus wird dabei auf klar umschriebene und eingrenzbare Ziele und auf die Umsetzung von definierten Massnahmen in den Betrieben gelegt, wie beispielsweise die betriebsinterne Wissensverbreitung oder das Training der konkreten Anwendung der Checkliste. Die definierten Ziele werden mittels spezifisch für dieses Programm ausgewählter und entwickelter Evaluationserhebungen überprüft. Die Wissensvermittlung und Einstellungen gegenüber der Checkliste, das Sicherheitsklima, der Umfang und die Qualität der Anwendung werden im Rahmen von progress! Sichere Chirurgie zu mehreren Zeitpunkten explizit evaluiert. Die Evaluation erfolgt einerseits auf Betriebsebene, damit betriebsintern der Stand der Umsetzung zurückgespiegelt werden kann - ein zentraler Faktor für das Erzielen erfolgreicher Veränderungsprozesse im Gesundheitswesen $[9,12]$. Andererseits sollen die Erkenntnisse über das gesamte Vertiefungsprojekt bei der Entwicklung weiterer Programme zur Erhöhung der Patientensicherheit in der Schweiz einfliessen können.

Die am Vertiefungsprojekt beteiligten Pilotbetriebe können von einem konzeptionell gut durchdachten Projekt [13] und dazu von verschiedenen Angeboten der Stiftung für Patientensicherheit profitieren. Ein zentrales Element sind die vier halbjährlich stattfindenden interprofessionellen und überbetrieblichen Workshops. Diese dienen dem Know-how Transfer und dem gegenseitigen interprofessionellen und überbetrieblichen Austausch. Mit der Vernetzung mehrerer Betriebe zum Wissensaustausch wird zudem ein gemeinsames Grundverständnis zur chirurgischen Checkliste und zum Wissen über deren Anwendung in der Schweiz geschaffen. Ein solches Grundverständnis ist bei der bestehenden hohen Fluktuation der Mitarbeitenden im Gesundheitswesen ein nicht zu vernachlässigender Punkt. www.patientensicherheit.ch

Stiftung für Patientensicherheit $(\mathrm{CH})$. Patientensicherheit in der Chirurgie. Anwendung und Akzeptanz chirurgischer Checklisten in der Schweiz. Ergebnisbericht. Zürich: Stiftung für Patientensicherheit; 2013.

Der Bericht kann bei der Stiftung für Patientensicherheit angefordert werden: info[at]patientensicherheit.ch

Detaillierte Informationen zur Intervention der chirurgischen Checkliste und zum Handlungsbedarf in der Schweiz wurden bereits Ende März 2013 in einem separaten Artikel beschrieben («Mühsam ernährt sich das Eichhörnchen» [10]).

\section{Im Vertiefungsprojekt soll die gewünschte Erhöhung der Compliance der korrekten Anwendung der chirurgischen Checkliste erreicht werden.}

allen Patienten unerlässlich. Das Vertiefungsprojekt progress! Sichere Chirurgie will deshalb dazu beitragen, dass die chirurgische Checkliste mit ihren drei Teilen [Sign In, Team Time Out und Sign Out] umfassend und korrekt umgesetzt wird ${ }^{3}$. Gerade das Erreichen einer 100\%-Anwendung ist eine grosse Herausforderung und erfordert einen entsprechenden Effort und spezielle Massnahmen.

Neben den schon aufbereiteten Empfehlungen und Hintergrundinformationen zur erfolgreichen Umsetzung der chirurgischen Checkliste in Form einer Schrift [11] soll nun im Vertiefungsprojekt in einem Verbund von ausgewählten Betrieben aus allen Landesteilen der Schweiz und mittels strukturiertem
Damit das gemeinschaftliche Vorgehen allen Betrieben auch den erwünschten Mehrnutzen bringt, wurden zudem Projektprämissen definiert, zu deren Respektierung sich alle Projektpartner mit der Unterzeichnung der Projektvereinbarung verpflichten. Dies sind beispielweise die betriebsinterne Veränderungsbereitschaft, die Ziel- und Faktenorientierung oder die Projektumsetzung mit einer interprofessionellen Projektgruppe oder die Teilnahme der betriebsinternen interprofessionellen Projektgruppen an den Workshops.

Weitere Faktoren, die bei der Implementierung von Massnahmen in einer komplexen Organisation relevant sind, wurden bei der Konzeptentwicklung 
des Vertiefungsprojektes progress! Sichere Chirurgie ebenfalls berücksichtigt:

- Die Direktion und das obere Kader verpflichten sich, sich explizit für das Projekt zu engagieren, indem sie die Projektvereinbarung mitunterzeichnen, die Ziele in die Unternehmensziele integrieren, die notwendigen Ressourcen zur Verfügung stellen oder sich auch an betriebsinternen Veranstaltungen engagieren.

- Das Gewinnen von Ambassadoren - in der Literatur auch Meinungsführer oder «Champions» genannt - ist gemäss diverser Studienergebnisse für den erfolgreichen Veränderungsprozess zentral [14]. Diese befürworten und fördern explizit die Verwendung der Checkliste. Sowohl auf nationaler Ebene wie betriebsintern werden ausgewählte anerkannte Personen diese Funktion übernehmen.

- Die chirurgische Checkliste wird betriebsintern an die lokalen Gegebenheiten angepasst. Alle Mitarbeitenden werden verbindlich mittels Wissensvermittlung über das «wie» und «warum» und mittels Training eingebunden. Es wird eine Kombination gewählt von klaren Richtlinien, einheitlichem Vorgehen und explizitem Einfordern der Massnahmen neben der Berücksichtigung der lokalen Gegebenheiten und Ausgangslagen wie der Organisationsstruktur oder -kultur [12].

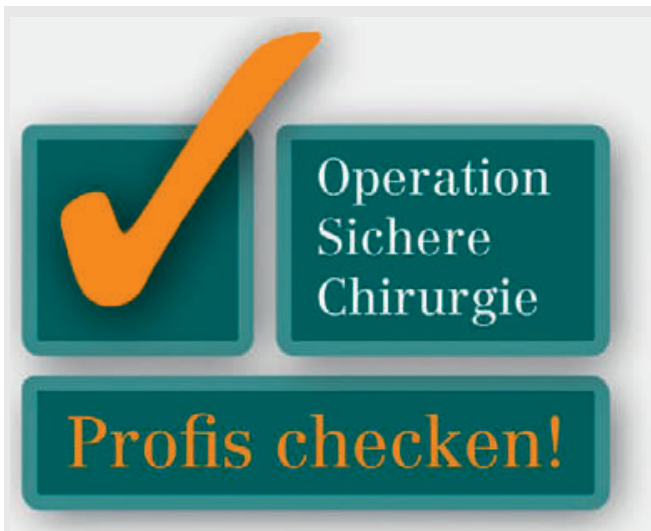

\section{Vertiefungsprojekt progress! Sichere Chirurgie der Stiftung für Patientensicherheit \\ Interessierte Betriebe können sich bis 3. Juni 2013 anmelden auf www.patientensicherheit.ch}

Das Pilotprogramm läuft unter dem Slogan «Operation Sichere Chirurgie Profis checken!». Die Grundlagen wurden in enger Kooperation mit zahlreichen Experten aus dem Schweizer Gesundheitswesen und den Fachgesellschaften der fmCh sowie den Berufsverbänden des weiteren OP- und Anästhesiepflegepersonals erarbeitet.

Kontakt: Stiftung für Patientensicherheit, Paula Bezzola, MPH, Programmleiterin und stv. Geschäftsführerin, Tel. 043244 14 80, bezzola[at]patientensicherheit.ch
Die Anzahl der Anmeldungen für das Vertiefungsprojekt progress! Sichere Chirurgie (vgl. Kasten) wird ein erster Hinweis sein, ob die Betriebe dieses neue Angebot mit einem interprofessionellen, betriebsübergreifenden und strukturierten Vorgehen nutzen wollen, um das Potential der chirurgischen Checkliste als wirksames und gleichzeitig effizientes und kostengünstiges Instrument zur Verbesserung der Patientensicherheit auszuschöpfen zu können.

\section{Literatur}

1 Pronovost P, Needham D, Berenholtz S, Sinopoli D, $\mathrm{Chu} \mathrm{H}$, Cosgrove $\mathrm{S}$, et al. An Intervention to Decrease Catheter-Related Bloodstream Infections in the ICU. N Engl J Med. 2006; 355(26):2725-32.

2 Haynes AB, Weiser TG, Berry WR, Lipsitz SR, Breizat AH, Dellinger EP, et al. A Surgical Safety Checklist to Reduce Morbidity and Mortality in a Global Population. N Engl J Med. 2009; NEJMsa0810119.

3 Perla RJ, Bradbury E, Gunther-Murphy C. Large-Scale Improvement Initiatives in Healthcare: A Scan of the Literature. J Healthc Qual. 2013;35(1):30-40.

4 Conley DM, Singer SJ, Edmondson L, Berry WR, Gawande AA. Effective surgical safety checklist implementation. J Am Coll Surg. 2011;212(5):873-9.

5 Schouten LMT, Hulscher MEJL, Everdingen JJE, Huijsman R, Grol RPTM. Evidence for the impact of quality improvement collaboratives: systematic review. BMJ. 2008; 336(7659):1491-4.

6 McCannon CJ, Perla RJ. Learning Networks for Sustainable, Large-Scale Improvement. Jt Comm J Quality safety. 2009;35:286-91.

7 Benn J, Burnett S, Parand A, Pinto A, Iskander S, Vincent C. Perceptions of the impact of a large-scale collaborative improvement programme: experience in the UK Safer Patients Initiative. J Eval Clin Pract. 2009;15(3):524-40.

8 Hulscher ME, Schouten LM, Grol RP, Buchan H. Determinants of success of quality improvement collaboratives: what does the literature show? BMJ Quality \& Safety. 2013;22(1):19-31.

9 Pronovost PJ, Berenholtz SM, Morlock LL. Is quality of care improving in the UK? BMJ 2011;342:c:6646.

10 Mascherek A, Bezzola P, Schwappach DLB. Mühsam ernährt sich das Eichhörnchen. Schweiz Ärztezeitung 2013;94(13/14):507-9.

11 Pezzola P, Hochreutener MA, Schwappach DLB. Operation Sichere Chirurgie. Die chirurgische Checkliste und ihre Implementierung: Kultur-TeamTools. Zürich: Stiftung für Patientensicherheit; 2012.

12 Healy JM. How hospital leaders implemented a safe surgery protocol in Australian hospitals. Int J Qual Health Care. 2012;24(1):88-94.

13 Goeschel CA, Weiss WM, Pronovost PJ. Using a logic model to design and evaluate quality and patient safety improvement programs. Int J Qual Health Care. 2012;24(4):330-7

14 Borchard A, Schwappach DLB, Barbir A, Bezzola P. A Systematic Review of the Effectiveness, Compliance, and Critical Factors for Implementation of Safety Checklists in Surgery. Ann Surg. 2012;256(6):925-33. 